Research Article

\title{
Cryosurgery as Additional Treatment in Tenosynovial Giant Cell Tumors
}

\author{
F. G. M. Verspoor, A. Scholte, I. C. M. van der Geest, G. Hannink, and H. W. B. Schreuder \\ Department of Orthopedics, Radboud University Medical Center, Nijmegen, Netherlands \\ Correspondence should be addressed to F. G. M. Verspoor; floorverspoor@gmail.com
}

Received 22 August 2016; Revised 1 November 2016; Accepted 4 December 2016

Academic Editor: R. Lor Randall

Copyright ( 2016 F. G. M. Verspoor et al. This is an open access article distributed under the Creative Commons Attribution License, which permits unrestricted use, distribution, and reproduction in any medium, provided the original work is properly cited.

\begin{abstract}
Introduction. Tenosynovial giant cell tumors (TGCT) emerge from the synovium and can behave aggressively. Surgical resection is the standard treatment. However, up to half of the patients with diffuse type show recurrences. Several additional treatments have been applied to reduce recurrences; none of these treatments was proven to be superior to surgical resection solely. This article describes the results of additional cryosurgery to surgical resection. Materials and Methods. We retrospectively evaluated 141 TGCT patients, between 1999 and 2007. Twelve patients had additional cryosurgery. The knee $(n=8)$, hip $(n=2)$, ankle $(n=1)$, and elbow $(n=1)$ were affected. Primary outcome variables were treatment indications, recurrences, and complications. Results. Indications for additional cryosurgery were extended disease, bone involvement, and locations that are difficult to surgically get disease-free such as cruciate ligaments. Five patients had recurrent disease, all of which had prior treatments. None of the primary treated patients had recurrent disease. One patient had a deep infection. Discussion. Cryosurgery may serve as an additional treatment for diffuse TCGT in selected cases. However, because of the small number of patients and the heterogeneous group we could not prove an advantage of additional cryosurgery over surgical resection only. Cryosurgery should be considered for further evaluation in a prospective study. If there is any effect it would be helpful, especially in patients with multiple TGCT recurrences.
\end{abstract}

\section{Introduction}

The tenosynovial giant cell tumor (TGCT), formerly known as pigmented villonodular synovitis (PVNS), is a rare proliferative disorder originating from the synovial membrane. Its annual incidence is described as 1.8 per million US citizens [1]. TGCT has a neoplastic origin, with a reactive component based on specific genetic changes [2,3]. Although TGCT can behave locally aggressive, it is considered a benign disorder [2]. Two subtypes have been described, (1) the less aggressive, localized form (LTGCT), which usually is a single nodular lesion, and (2) the diffuse form (DTGCT), which usually affects the whole joint and frequently recurs $(14-55 \%)[4,5]$. The knee $(75 \%)$ is the most frequently affected joint, followed by the hip, ankle, elbow, and other synovial joints [2].

The recommended treatment for TGCT is surgical resection of all affected tissue [4]. Incomplete resection or multiple treatments can result in functional impairment [6]. With extended or recurrent disease, several additional treatments (e.g., External Beam Radiotherapy (EBRT), radiosynovectomy, and immune- and targeted therapy) have been applied to reduce recurrence rates [4]. None of these treatments was proven to be superior to surgical resection on its own, which makes aggressive, persistently recurrent TGCT difficult to cure [4].

Cryosurgery, in the form of liquid nitrogen spray, is widely used as an additional treatment for various bone tumors [7]. In order to try to reduce recurrence rates, we started using cryosurgery as an additive treatment to surgical resection of TGCT in selected cases. Mohler and Kessler [8] reported on the successful treatment of three TGCT patients through surgical synovectomy combined with cryosurgery. To the best of our knowledge, there are no other publications that describe cryosurgery as an additive treatment to surgical resection, in the treatment of TGCT.

We retrospectively evaluated TGCT patients treated with cryosurgery in addition to surgical synovectomy, with respect 
to the indication for additional treatment(s), the number of recurrences, and complications.

\section{Materials and Methods}

We retrospectively searched pathologic and radiologic reports in the hospital database with the keywords "pigmented villonodular synovitis", "local tenosynovitis", and "giant cell tumor of the tendon sheath". Between 1985 and 2014, 141 TGCT patients were identified; 48 (34\%) with localized and $93(66 \%)$ with diffuse disease. According to operative reports, cryosurgery was used in 12 patients, between 1999 and 2007. Clinical, pathological, radiological, treatment, and follow-up information was obtained from medical records. The study protocol (CMO2015-1915) was assessed by our institutional review board (the research ethics committee of the Radboud University Nijmegen Medical Center) and was carried out in Netherlands in accordance with the applicable rules concerning the review of research ethics committees and informed consent.

There was no protocol for the use of additional cryosurgery. All patients with extended or recurrent disease were discussed in a multidisciplinary team (medical oncology, radiotherapy, surgical oncology, and orthopedic surgical oncology). However, eventually it was the surgeon's call for each individual case whether or not to use cryosurgery. In the 12 patients who received additional cryosurgery the knee $(n=8)$, hip $(n=2)$, ankle $(n=1)$, and elbow $(n=1)$ were affected. All diagnoses were histologically confirmed. One patient (elbow) had localized disease; all other patients had diffuse disease. The mean age at the time of cryosurgery was 39 (range 21-61) years. All patient characteristics are listed in Table 1.

Cryosurgery was used in addition to a surgical synovectomy. After complete removal of the affected tissue, the noncartilaginous surface of the joint (synovium, joint capsule, and bone) was treated with three cycles of liquid nitrogen spray. All frozen tissue got to at least $-50^{\circ} \mathrm{C}$. Spontaneous thawing warmed the tissue up to $20^{\circ} \mathrm{C}$. After three cycles of rapid cooling and spontaneous thawing the entire wound was lavaged with sodium hyponitrite to prevent seeding of tumor cells. A healthy circulation (no use of a tourniquet) is important to protect neurovascular structures and the skin $[7,9]$. To the best of our knowledge there is no literature available yet on costs of cryosurgical treatment. However, we would estimate a maximal extra surgical time of 15 minutes (i.e., three times 5 minutes, quick freeze, and slow thawing). The materials needed for cryosurgery are a little container and temperature sensors. Liquid nitrogen costs 10 euro cents a liter and we use less than a liter for one patient. Details of all received treatments are listened in Table 1.

Between 1985 and 2014 our follow-up protocol has changed from an intensive follow-up with 3 monthly visits, including a MRI every 6 months, to only a single clinical follow-up visit 3 months postoperatively with additional visits when clinical symptoms appear, a "wait and see policy."

Outcomes were the indications for additional cryosurgery, recurrent disease, and complications. To be able to formulate indications for cryosurgery we scored extra-articular disease, large expansion (described in operative reports as extensive or bulky disease or when two or more compartments of the knee were involved), bone involvement, and involvement of the cruciate ligaments. Local recurrent disease was defined as histological proven recurrent disease.

Descriptive statistics were used to summarize the data. No further analyses were performed because of the small number and diversity of patients.

\section{Results}

Of the 141 identified TGCT patients, 12 patients received additional cryosurgery. In the 129 patients without additional cryosurgery, 82 had diffuse disease. Fourteen of these patients had no treatment at our tertiary center. In 68 patients treated at our center the knee $(n=59)$, hip $(n=3)$, ankle $(n=5)$, and elbow $(n=1)$ were affected. Three patients were lost to follow-up. The mean time from first treatment to follow-up was 10 (range $0.6-41$ ) years.

3.1. Indications for Cryosurgery. In the 12 cryosurgical patients, five had primary disease and seven had prior treatments (1-3 previous treatments). The mean time from diagnose to cryosurgical treatment was 4.5 (range 0.5-6.3) years. In 10 out of 12 patients, the entire intra-articular space was affected with extension in surrounding structures, such as bone, ligaments, fat, and muscle tissue. All were of diffuse type TGCT. Seven of these patients had extra-articular disease. The cruciate ligaments of the knee were involved in 6 out of 8 patients. In 7 out of 12 patients ( $n=4$ knees, $n=2$ hips, and $n=1$ ankle) there was bone involvement. Bone involvement and extended disease were reported to be indications for additional cryosurgery to surgical synovectomy.

In one patient with localized disease of the elbow, we retrospectively did not find the considerations for additional cryosurgery. Probably the surgeon wanted to minimize the chance on recurrent disease in this location.

In patients with DTGCT of the knee $(n=59), 19$ patients had extra-articular disease, 4 patients had bone involvement, 52 patients had intracondylar involvement, and 55 had extended disease or a combination of two, three, or all four of these features. All five ankles had extended disease with extraarticular involvement, and one of these patients also had bony involvement.

3.2. Recurrences. The mean follow-up after cryosurgery was 7.7 (range 5.5-14.9) years. Five patients with the diffuse subtype developed recurrent disease after cryosurgery, at a mean time of 2.8 (range 0.7-9.0) years. All recurrences were diagnosed on MRI, which was performed when clinical symptoms of any kind in or nearby the affected joint presented. Four were also histologically confirmed. None of these five patients had primary disease; they already had recurrent disease at the time of cryosurgical therapy. One patient with recurrent disease received EBRT following initial cryosurgery. Another patient received a total hip arthroplasty (THA) during the same surgical procedure. 
TABLE 1: Demographics, previous recurrences, and detailed description of treatments received before or in combination with additional cryosurgery.

\begin{tabular}{|c|c|c|c|c|c|c|c|c|c|}
\hline \multirow{2}{*}{$\begin{array}{l}\text { Patient } \\
\text { ID }\end{array}$} & \multirow{2}{*}{ Sex } & \multirow{2}{*}{$\begin{array}{l}\text { Age (at time of } \\
\text { surgery) }\end{array}$} & \multirow{2}{*}{ Localized/diffuse } & \multirow{2}{*}{$\begin{array}{l}\text { Previous } \\
\text { recurrences }\end{array}$} & \multirow{2}{*}{$\begin{array}{l}\text { Follow-up before } \\
\text { cryosurgery (years) }\end{array}$} & \multicolumn{4}{|c|}{ All treatments received } \\
\hline & & & & & & 1st & 2nd & $3 \mathrm{rd}$ & 4 th \\
\hline \multicolumn{10}{|l|}{ Knee } \\
\hline 1 & $\mathrm{~F}$ & 61 & Dt-GCT & 3 & 6.3 & SE & SE & $\mathrm{tSE}$ & $\begin{array}{l}t S E+\text { cryo } \\
+E B R T\end{array}$ \\
\hline 2 & M & 58 & Dt-GCT & 2 & 9.7 & Yt & SE & $\begin{array}{l}t S E+\text { cryo } \\
+E B R T\end{array}$ & \\
\hline 3 & F & 46 & Dt-GCT & 2 & 3.2 & SE & SE & $S E+$ cryo & \\
\hline 4 & $\mathrm{M}$ & 51 & Dt-GCT & 0 & 0.3 & $t S E+c r y o$ & & & \\
\hline 5 & $\mathrm{~F}$ & 25 & Dt-GCT & 1 & 3.0 & tSE & $S E+$ cryo & & \\
\hline 6 & $\mathrm{~F}$ & 49 & Dt-GCT & 0 & 0.1 & $S E+$ cryo & & & \\
\hline 7 & M & 31 & Dt-GCT & 2 & 4.9 & tSE & SE & $S E+$ cryo & \\
\hline 8 & $\mathrm{~F}$ & 31 & Dt-GCT & 0 & 0.0 & $t S E+c r y o$ & & & \\
\hline \multicolumn{10}{|l|}{ Hip } \\
\hline 9 & M & 44 & Dt-GCT & 2 & 0.5 & $\mathrm{tSE}$ & SE & $\begin{array}{l}S E+T H A \\
\quad+\text { cryo }\end{array}$ & \\
\hline 10 & $\mathrm{~F}$ & 20 & Dt-GCT & 2 & 4.0 & SE & Yt & $\begin{array}{l}S E+T H A \\
\quad+\text { cryo }\end{array}$ & \\
\hline \multicolumn{10}{|l|}{ Ankle } \\
\hline 11 & M & 34 & Dt-GCT & 0 & 0.0 & $S E+$ cryo & & & \\
\hline \multicolumn{10}{|l|}{ Elbow } \\
\hline 12 & $\mathrm{~F}$ & 21 & Lt-GCT & 0 & 0.7 & $S E+$ cryo & & & \\
\hline
\end{tabular}

F: female, M: male, SE: surgical synovectomy, tSE: two-stage synovectomy (anterior and posterior compartment of the knee in two separate procedures), Yt: yttrium radiosynovectomy, EBRT: external beam radiation therapy, cryo: cryosurgery, follow-up before cryosurgery: the time from diagnose until the use of cryosurgery in years, and THA: total hip arthroplasty.

Three out of five patients with recurrent disease after cryosurgery developed a second or even third recurrence. Treatments following these recurrences are listed in Table 2.

All four recurrences in patients with TGCT of the knee occurred within 2 years (9-22 months) after treatment. These patients had extended disease with involvement of the cruciate ligaments. In two patients with recurrent disease there was extra-articular disease and in one there was bone involvement. In the four knee patients without recurrences (of whom three were primary treated and one was treated with additional EBRT following cryosurgery), three had extended disease with bone involvement.

In the two patients with TGCT of the hip, one recurrence occurred nine years after cryosurgery. It was the third recurrence since onset of disease. At the time of cryosurgery, the acetabulum was affected by TGCT. Both patients also received a total hip arthroplasty for joint destruction by extended disease combined with secondary osteoarthritis. In both ankle and elbow, no recurrences were seen.

In patients with diffuse type disease whom did not receive additional cryosurgery $30(44 \%)$ had recurrent disease. The mean time to first recurrence was 4.7 (range 0.7-27) years. All referred knee patients $(n=26)$ had recurrent disease. Nine $(34.6 \%)$ of these patients developed another recurrence after treatment at our center in 1.7 (range 0.7-4.4) years. In primary treated knee patients $16(55 \%)$ had recurrent disease in 6.4 (range 1-27) years.

Twenty-seven patients (40\%) had extra-articular disease, 6 (9\%) had bone involvement, 52 (77\%) had involvement of the cruciate ligaments, and in $64(94 \%)$ patients there was extended disease or a combination of two, three, or all four of these features. For example, in the 27 patients with extra-articular disease 6 had bone involvement, 18 had affected cruciate ligaments, and all 27 had extended disease. Eighteen of these patients had recurrent disease in 7 ( 0.8 17) years of follow-up. The time to first recurrence was 1.9 $(0.7-4.1)$ years. And 11 recurrences appeared after treatment at our center. All 6 patients with bone involvement had extraarticular disease and extended disease, including 4 patients with affected cruciate ligaments. Five of these patients had recurrent disease in $11(2-17)$ years of follow-up. The time to first recurrence was $1.8(1.2-3.2)$ years. Three recurrences appeared after treatment at our center.

Of five patients with TGCT in the ankle 4 had recurrent disease, two of which were primary treated patients. The mean time until first recurrence was 2.7 (1.2-4.1) years.

3.3. Complications. Two complications were reported in the cryosurgical group (Table 2): a hip dislocation direct after 
4

Sarcoma

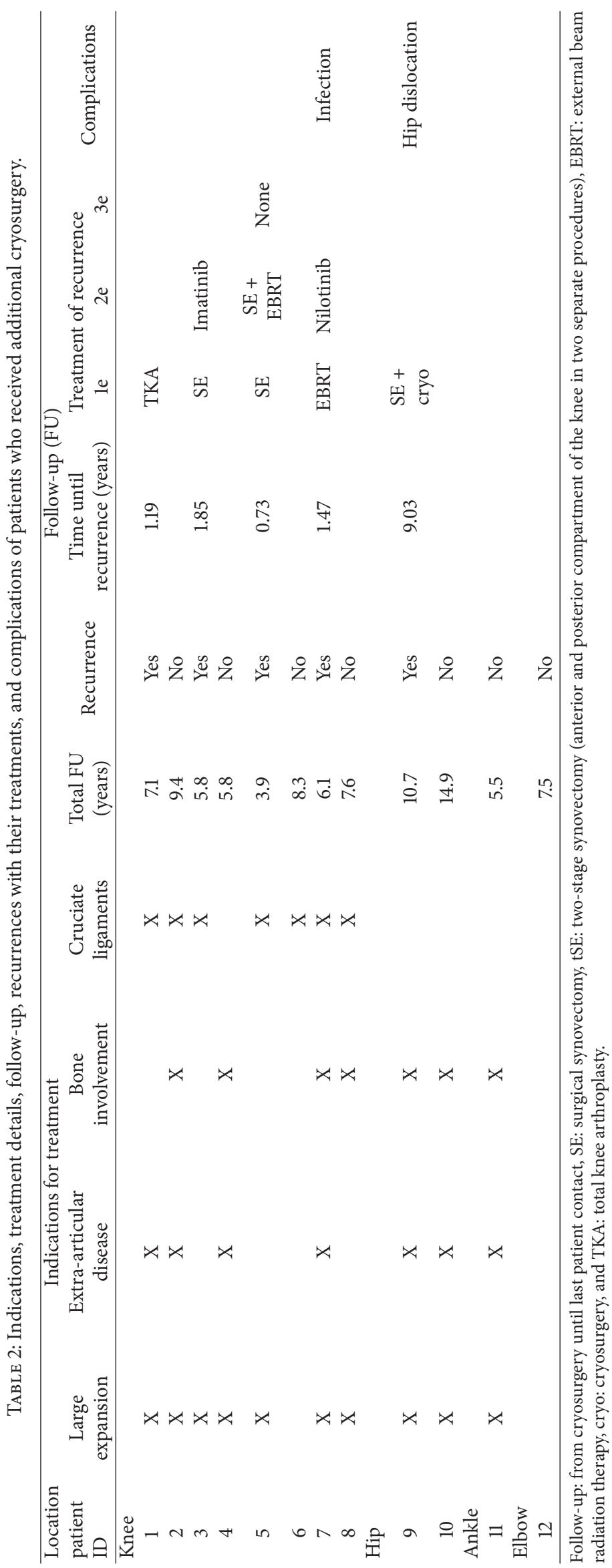


arthroplasty, which was closed reduced, and a deep infection of the knee, three months after surgery, which was treated with surgical debridement and antibiotics.

\section{Discussion}

TGCT is difficult to cure; in particular, the diffuse subtype frequently recurs. The treatment of first choice is surgical resection of all affected tissue. However, with extended and/or extra-articular disease, bone involvement, or disease at key locations inside the joint (ACL, PCL, and collateral ligaments), resection of all affected tissue can be difficult to achieve. Various treatments (e.g., radiosynovectomy, EBRT, and immune- or targeted treatment) have been used, without the desired results [4]. We retrospectively evaluated the effect of additional cryosurgery in the treatment of TGCT.

We found that indications of cryosurgery in addition to surgical resection for TGCT were the presence of extended, extra-articular disease, affected bone, or locations surgically difficult to get disease-free, such as the cruciate ligaments. Furthermore, no recurrent disease was observed in patients primary treated with surgical synovectomy combined with cryosurgery. In patients known with recurrent disease additional cryosurgery was not effective.

Various indications were described for additional therapies in the treatment of TGCT [4]. The indications found for cryosurgery were similar to those considered for EBRT, except for patients with involvement of the neurovascular bundle. Cryosurgery is contraindicated in these patients. Effectiveness of radiosynovectomy in the treatment of TGCT is not proven and does not cover the extra-articular space [5]. Therefore, it is not indicated in patients with extended disease. Immunotherapy is not validated yet for the treatment of TGCT; however it has been applied in patients with extended disease $[10,11]$. For the use of targeted therapy in the treatment of TGCT, only small case studies and one retrospective cohort (29 patients) are published so far [3, 1214]. Due to the rapid relapse after discontinuation and severe side effects of targeted therapy, these treatments are only recommended for patients with surgical unresectable disease or multiple recurrences that produce clinical and functional impairment [12]. Other systemic treatments are individually available in trial settings [15].

Additional cryosurgery was not used after 2007 for different reasons: less extreme TGCT cases were presented the last years. The surgeon who often used cryosurgery for TGCT retired. Others preferred a more throughout resection of affected tissue. There were definitely no adverse effects of cryosurgery that made us stop using it.

The number of recurrences in DTGCT with or without the use of cryosurgery were similar, respectively, $45 \%$ after 2.8 years and $44 \%$ after 4.7 years. However, retrospectively it is difficult to determine if the patients in the cryosurgical group had worse disease compared to the other patients. In the cryosurgical group there was $90 \%$ extended disease, $64 \%$ extra-articular disease, and $64 \%$ bone involvement and in $88 \%$ the cruciate ligaments were involved. The others had 94\% extended disease, $40 \%$ extra-articular disease, $6 \%$ bone involvement, and $77 \%$ involvement of the cruciate ligaments.
In both groups there was a great difference in recurrences between referred patients and primary treated patients. There were five $(71 \%)$ recurrences after cryosurgery, in patients with prior treatments. Two out of seven patients with bone involvement had recurrent disease. None of the primary treated TGCT patients had recurrences after surgical synovectomy with cryosurgery. In the patients not treated with cryosurgery all referred knee patients showed recurrent disease of which $35 \%$ had recurrent disease after treatment at our center. There were $55 \%$ recurrences in the primary treated knee patients.

Mohler and Kessler [8] described the use of cryosurgery in addition to surgical synovectomy in three patients (25-31 years of age) with TGCT of the knee. Two of these patients were diagnosed with recurrent disease. Cryosurgery was used during their third treatment. The other patient had primary disease. Two patients had extended disease. But, no details of bone involvement or involvement of the cruciate ligaments were described. No recurrent disease was found after 1431 months of follow-up. However, TGCT recurrences are described up to 14 years later [16].

Although cryosurgery is uncommon in the treatment of TGCT, it has been described for orthopedic oncologic diseases [7]. Reported complications as a result of cryosurgery are postoperative fractures (5-30\%), infections (4\%), transient nerve palsies, and gas-embolisms (0.3\%) [7]. Also, damage to surrounding structures, such as the epiphysis and cartilage, has been described. In case of TGCT, the chance on fractures will be negligible though the intraarticular location. However, for this reason there might be an increased risk on damage to the joint cartilage. Furthermore, in TGCT the cartilage is often already damaged due to the disease itself or to the multiple prior treatments. None of these complications were found in this study. However, one patient with recurrent disease after cryosurgery received a total knee arthroplasty for secondary osteoarthritis. Also, a postoperative dislocation of the hip and a deep infection of the knee three months after surgery occurred. These could be general complications after surgery; however, increased risk on infections have been described for cryosurgery [7].

\section{Abbreviations}

TGCT: Tenosynovial giant cell tumor

LTGCT: Localized tenosynovial giant cell tumor

DTGCT: Diffuse tenosynovial giant cell tumor

EBRT: External beam radiation therapy

THA: Total hip arthroplasty

TKA: Total knee arthroplasty.

\section{Additional Points}

Limitations. It is very difficult to produce a large volume study on treatment effects in a rare disease such as TGCT. For the subgroup of patients with extended disease, this is even more difficult. Therefore the study is limited by its retrospective character and the small number of patients. However, detailed information on received treatments was available and carefully described. Furthermore, some patients 
had other additional treatments next to cryosurgery, such as EBRT or arthroplasty what could have influenced our results. However, this was seen in both patient groups with and without recurrent disease. Despite these limitations, this article provides cautious insight for a possible role of cryosurgery in the treatment of TGCT. A prospective study with clear indications should follow to show if there is a role for cryosurgery in a subgroup of TGCT patients. We conclude that cryosurgery may serve as an additional treatment for diffuse TCGT in selected cases. However, because of the small number of patients and the heterogeneous group we could not prove an advantage of additional cryosurgery over surgical resection only. Cryosurgery is a cheap, easy, and relative harmless technique that should be considered for further evaluation in a prospective study with a sufficiently large number of patients, clear indications, and a proper control group. If there is any effect it would be helpful, especially in patients with multiple TGCT recurrences.

\section{Competing Interests}

None author (F. G. M. Verspoor, G. Hannink, A. Scholte, I. C. M. van der Geest, or H. W. B. Schreuder) or any member of his or her immediate family has funding or commercial associations (e.g., consultancies, stock ownership, equity interest, and patent/licensing arrangements) that might pose a conflict of interests in connection with the submitted article.

\section{Authors' Contributions}

The study concept and design were developed by F. G. M. Verspoor, I. C. M. van der Geest, and H. W. B. Schreuder. A. Scholte and F. G. M. Verspoor carried out the data acquisition. The quality control of data and algorithms were carried out by F. G. M. Verspoor and H. W. B. Schreuder. F. G. M. Verspoor and G. Hannink did the data analysis, interpretations, and statistical analysis. The manuscript preparation and editing was done by F. G. M. Verspoor and A. Scholte. All authors (F. G. M. Verspoor, A. Scholte, I. C. M. van der Geest, G. Hannink, and H. W. B. Schreuder) reviewed and approved the final manuscript.

\section{References}

[1] B. W. Myers, A. T. Masi, and S. L. Feigenbaum, "Pigmented villonodular synovitis and tenosynovitis: a clinical epidemiologic study of 166 cases and literature review," Medicine, vol. 59, no. 3, pp. 223-238, 1980.

[2] C. D. M. Fletcher, K. K. Unni, and F. Mertens, Eds., Pathology and Genetics of Tumours of Soft Tissue and Bone, International Agency for Research on Cancer Press, Lyon, France, 2002.

[3] R. B. West, B. P. Rubin, M. A. Miller et al., "A landscape effect in tenosynovial giant-cell tumor from activation of CSF1 expression by a translocation in a minority of tumor cells," Proceedings of the National Academy of Sciences of the United States of America, vol. 103, no. 3, pp. 690-695, 2006.

[4] F. G. M. Verspoor, I. C. M. van der Geest, E. Vegt, R. P. H. Veth, W. T. van der Graaf, and H. W. B. Schreuder, "Pigmented villonodular synovitis: current concepts about diagnosis and management," Future Oncology, vol. 9, no. 10, pp. 1515-1531, 2013.

[5] S. Ottaviani, X. Ayral, M. Dougados, and L. Gossec, "Pigmented villonodular synovitis: a retrospective single-center study of 122 cases and review of the literature," Seminars in Arthritis and Rheumatism, vol. 40, no. 6, pp. 539-546, 2011.

[6] C. Chiari, C. Pirich, W. Brannath, R. Kotz, and K. Trieb, "What affects the recurrence and clinical outcome of pigmented villonodular synovitis?" Clinical Orthopaedics and Related Research, no. 450, pp. 172-178, 2006.

[7] R. Veth, B. Schreuder, H. van Beem, M. Pruszczynski, and J. de Rooy, "Cryosurgery in aggressive, benign, and low-grade malignant bone tumours," Lancet Oncology, vol. 6, no. 1, pp. 2534, 2005.

[8] D. G. Mohler and B. D. Kessler, "Open synovectomy with cryosurgical adjuvant for treatment of diffuse pigmented villonodular synovitis of the knee," Bulletin: Hospital for Joint Diseases, vol. 59, no. 2, pp. 99-105, 2000.

[9] H. W. B. Schreuder, J. Van Egmond, H. B. H. Van Beem, and R. P. H. Veth, "Monitoring during cryosurgery of bone tumors," Journal of Surgical Oncology, vol. 65, no. 1, pp. 40-45, 1997.

[10] L. van der Heijden, M. J. L. Mastboom, P. D. S. Dijkstra, and M. A. J. van de Sande, "Functional outcome and quality of life after the surgical treatment for diffuse-type giant-cell tumour around the knee: a retrospective analysis of 30 patients," Bone and Joint Journal, vol. 96, no. 8, pp. 1111-1118, 2014.

[11] J. Bickels, J. Isaakov, Y. Kollender, and I. Meller, "Unacceptable complications following intra-articular injection of yttrium 90 in the ankle joint for diffuse pigmented villonodular synovitis," Journal of Bone and Joint Surgery-Series A, vol. 90, no. 2, pp. 326-328, 2008.

[12] P. A. Cassier, S. Stacchiotti, H. Gelderblom et al., "Imatinib mesylate for the treatment of locally advanced and/or metastatic pigmented villonodular synovitis/tenosynovial giant cell tumor (PVNS/TGCT)," Journal of Clinical Oncology Conference, vol. $28,2010$.

[13] J.-Y. Blay, H. El Sayadi, P. Thiesse, J. Garret, and I. Ray-Coquard, "Complete response to imatinib in relapsing pigmented villonodular synovitis/tenosynovial giant cell tumor (PVNS/TGCT)," Annals of Oncology, vol. 19, no. 4, pp. 821-822, 2008.

[14] W. M. Snoots, D. Watkins, D. Dockery, R. Mennel, and B. S. Cheek, "Pigmented villonodular synovitis responsive to imatinib therapy," Baylor University Medical Center Proceedings, vol. 24, pp. 134-138, 2011.

[15] E. L. Staals, S. Ferrari, D. M. Donati, and E. Palmerini, "Diffusetype tenosynovial giant cell tumour: current treatment concepts and future perspectives," European Journal of Cancer, vol. 63, pp. 34-40, 2016.

[16] L. Vastel, P. Lambert, G. De Pinieux, O. Charrois, M. Kerboull, and J.-P. Courpied, "Surgical treatment of pigmented villonodular synovitis of the hip," Journal of Bone and Joint Surgery - Series A, vol. 87, no. 5, pp. 1019-1024, 2005. 


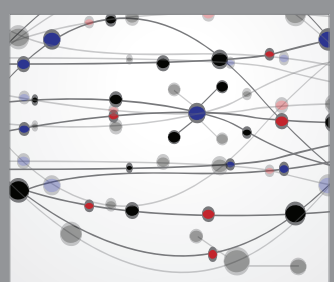

The Scientific World Journal
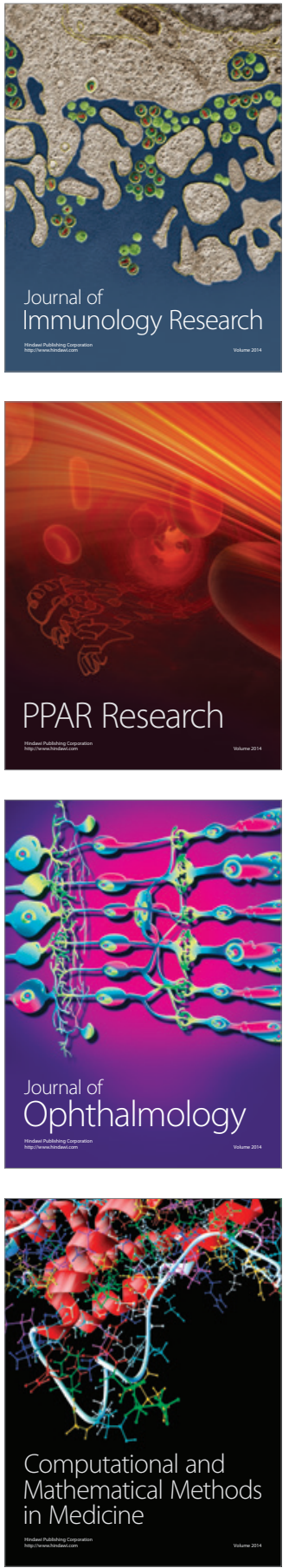

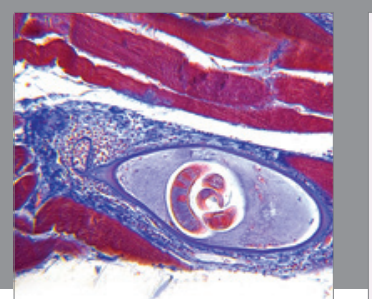

Gastroenterology Research and Practice

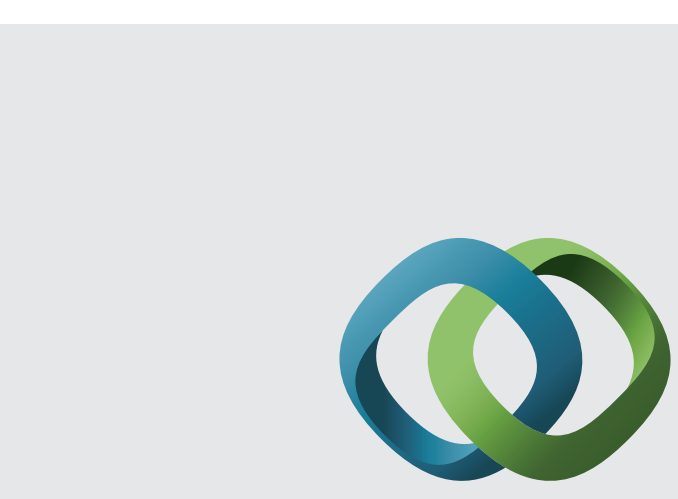

\section{Hindawi}

Submit your manuscripts at

http://www.hindawi.com
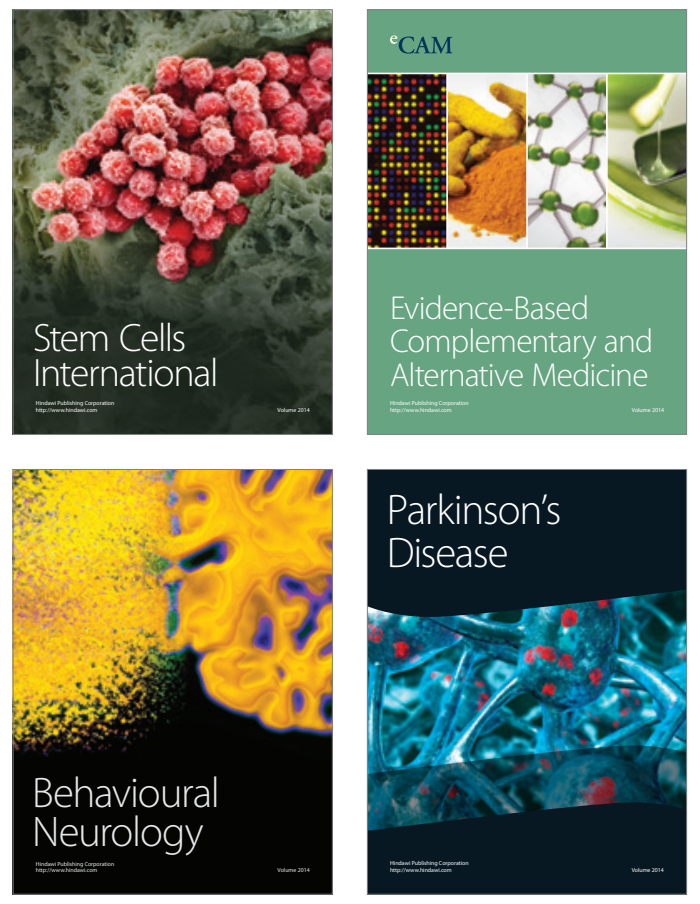
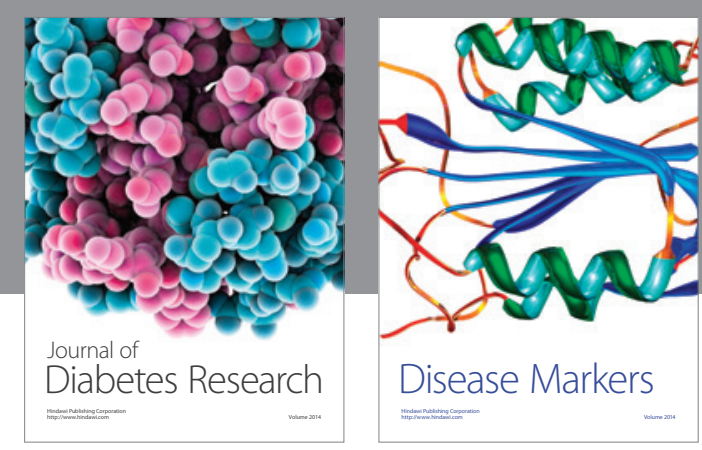

Disease Markers
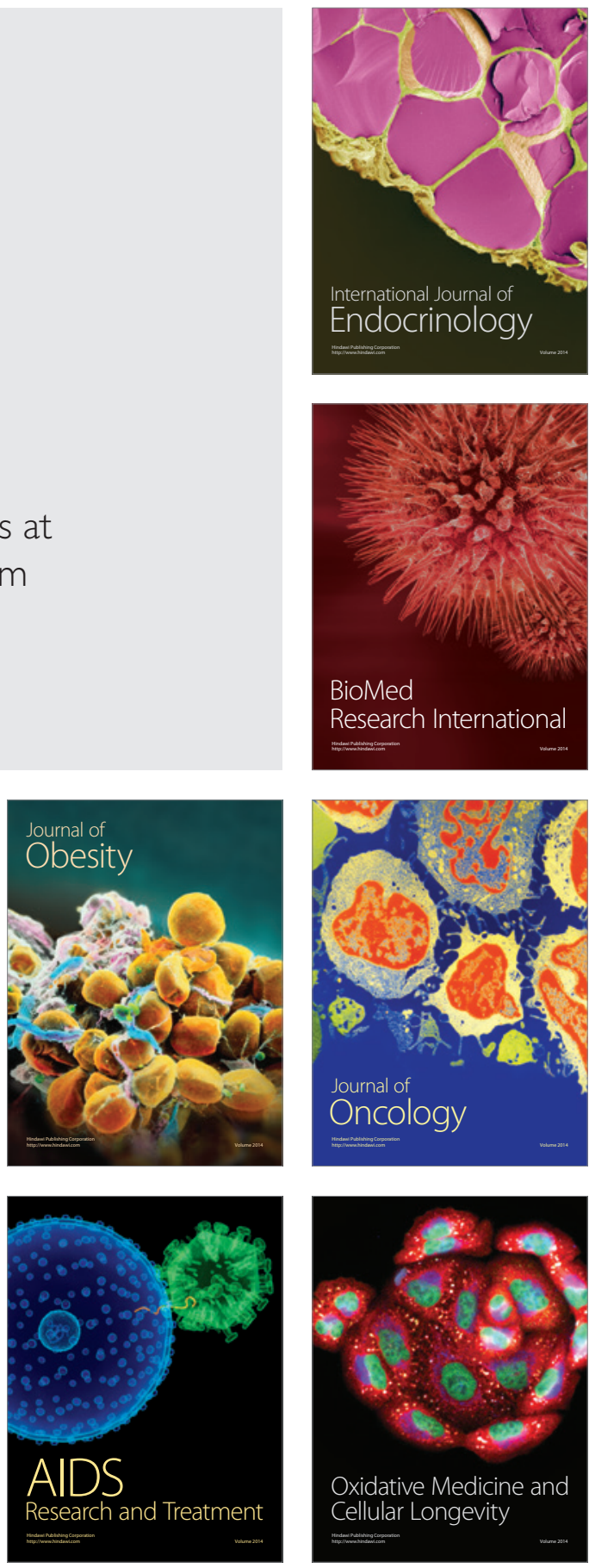